Single dose of mirtazapine modulates whole-brain functional connectivity during emotional narrative processing

\title{
Komulainen, Emma
}

2017-05-30

Komulainen, E, Glerean , E, Meskanen, K, Heikkila , R, Nummenmaa , L, Raij , T, Lahti , J , Jylhä , P , Melartin , T , Isometsa , E \& Ekelund, J 2017 , ' Single dose of mirtazapine modulates whole-brain functional connectivity during emotional narrative processing ' , Psychiatry Research. Neuroimaging , vol. 263 , pp. 61-69 . https://doi.org/10.1016/j.pscychresns.2017.03.009

http://hdl.handle.net/10138/236231

https://doi.org/10.1016/j.pscychresns.2017.03.009

unspecified

publishedVersion

Downloaded from Helda, University of Helsinki institutional repository.

This is an electronic reprint of the original article.

This reprint may differ from the original in pagination and typographic detail.

Please cite the original version. 


\title{
Single dose of mirtazapine modulates whole-brain functional connectivity during emotional narrative processing
}

\author{
Emma Komulainen $^{\mathrm{a}, *}$, Enrico Glerean ${ }^{\mathrm{b}}$, Katarina Meskanen $^{\mathrm{a}}$, Roope Heikkiläa \\ Lauri Nummenmaa ${ }^{c}$, Tuukka T. Raij ${ }^{\mathrm{a}, \mathrm{b}, \mathrm{d}}$, Jari Lahti ${ }^{\mathrm{e}, \mathrm{f},}$, Pekka Jylhä ${ }^{\mathrm{a}, \mathrm{h}}$, Tarja Melartin ${ }^{\mathrm{a}}$, \\ Erkki Isometsä ${ }^{\mathrm{a}}$, Jesper Ekelund ${ }^{\mathrm{a}, \mathrm{i}}$ \\ a University of Helsinki and Helsinki University Hospital, Psychiatry, Helsinki, Finland \\ b Aalto University, School of Science, Department of Neuroscience and Biomedical Engineering, Espoo, Finland \\ c Turku PET Centre and Department of Psychology, University of Turku, Turku, Finland \\ d Aalto NeuroImaging, Aalto University, Espoo, Finland \\ e University of Helsinki, Institute of Behavioral Sciences, Helsinki, Finland \\ ${ }^{\mathrm{f}}$ Folkhälsan Research Center, Helsinki, Finland \\ ${ }^{\mathrm{g}}$ Helsinki collegium of Advanced Studies, University of Helsinki, Finland \\ ${ }^{\mathbf{h}}$ National Institute of Health and Welfare, Department of Mental Health and Substance Abuse Services, Helsinki, Finland

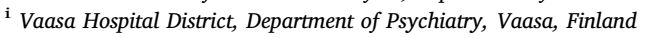

\section{A R T I C L E I N F O}

\section{Keywords:}

Antidepressant

fMRI

Emotion

Information processing

Neural network

\begin{abstract}
A B S T R A C T
The link between neurotransmitter-level effects of antidepressants and their clinical effect remain poorly understood. A single dose of mirtazapine decreases limbic responses to fearful faces in healthy subjects, but it is unknown whether this effect applies to complex emotional situations and dynamic connectivity between brain regions. Thirty healthy volunteers listened to spoken emotional narratives during functional magnetic resonance imaging (fMRI). In an open-label design, 15 subjects received $15 \mathrm{mg}$ of mirtazapine two hours prior to fMRI while 15 subjects served as a control group. We assessed the effects of mirtazapine on regional neural responses and dynamic functional connectivity associated with valence and arousal. Mirtazapine attenuated responses to unpleasant events in the right fronto-insular cortex, while modulating responses to arousing events in the core limbic regions and the cortical midline structures (CMS). Mirtazapine decreased responses to unpleasant and arousing events in sensorimotor areas and the anterior CMS implicated in self-referential processing and formation of subjective feelings. Mirtazapine increased functional connectivity associated with positive valence in the CMS and limbic regions. Mirtazapine triggers large-scale changes in regional responses and functional connectivity during naturalistic, emotional stimuli. These span limbic, sensorimotor, and midline brain structures, and may be relevant to the clinical effectiveness of mirtazapine.
\end{abstract}

\section{Introduction}

To develop more specific and efficient interventions for depression, understanding the brain network level mechanisms of action of available, effective medications would be valuable. Since the pathogenesis of depression is understood as dysfunctions of large-scale brain circuitries involved in emotional and cognitive processes (Kaiser et al., 2015), using complex and naturalistic stimuli and taking into account not only regional, but also network-level effects, could provide new insights about the effects of antidepressants on these circuitries. Antidepressants have been shown to influence resting state functional connectivity (Li et al., 2013), but their effect on large-scale functional connectivity during emotional processing remains unresolved. Antidepressants influence emotional processing, e.g. decreasing limbic responses to negative stimuli, as early as a few hours or days after administration (Harmer and Cowen, 2013). Previous studies have quantified the effect of antidepressants on brain responses to briefly flashed emotional stimuli, most commonly emotional facial expressions (e.g. (Harmer et al., 2006; Norbury et al., 2007), see Ma (2015) for meta-analysis). Although facial expressions are important in social interaction and interpreting the emotions of others, they provide a simplified model for emotional responses, lacking information about the dynamic nature of real-life information processing.

Storytelling is a fundamental means of natural human social

\footnotetext{
* Corresponding author.

E-mail address: emma.komulainen@helsinki.fi (E. Komulainen).
} 
interaction occurring in the forms of face-to-face communication, TV, radio, and movies. It is also a powerful way of eliciting strong emotional sensations; interpreting the emotions of others and sharing one's emotions via spoken and written narratives are crucial for social interaction. Therefore, auditory narratives provide a powerful model for daily emotional responses. Emotional stories activate the brain's emotional circuits similarly to simple visual emotional stimuli (Ferstl et al., 2005; Wallentin et al., 2011), consequently triggering changes in sensorimotor and visceral systems (Vrana and Lang, 1990), enabling humans to extract the emotional content from spoken or written stories. Recently, the networks of brain areas related to sound processing, language comprehension, and emotional and self-referential processing have been shown to become synchronized across healthy persons listening to emotional, spoken narratives (Nummenmaa et al., 2014b). Moreover, similarity in brain activity between subjects is associated with similarity in experienced emotional state (Nummenmaa et al., 2014b). Importantly, spoken narratives elicit strong, time-variable emotional reactions, the time series of which can be accurately tracked. They can thus be used for modeling the effects of emotion states on large-scale, dynamic connectivity changes.

Several monoaminergic antidepressants rapidly improve recognition of happy facial expressions in healthy volunteers (Harmer et al., 2003a, 2008, 2003b; Murphy et al., 2009) (but see also Browning et al. (2007), Harmer et al. (2004) for negative results). At the neural level, antidepressants decrease amygdala responses to negative stimuli (Anderson et al., 2007; Del-Ben et al., 2005; Murphy et al., 2009; Takahashi et al., 2005), even though increased responses (Bigos et al., 2008) and null effects (Norbury et al., 2009) have been also reported. Antidepressant effects elsewhere in the brain are less consistent, but decreased responses to negative stimuli in healthy volunteers have been reported in the ventrolateral prefrontal cortex (VLPFC) (Del-Ben et al., 2005; Takahashi et al., 2005), medial prefrontal cortex (MPFC) (Grady et al., 2013; Harmer et al., 2006), and striatum (Grady et al., 2013; Takahashi et al., 2005). These early changes counteract the negative information processing bias in depressed patients, a core factor in the pathogenesis of depression (Disner et al., 2011; Gotlib and Joormann, 2010), and may be essential for the sustained therapeutic effect of antidepressants. Indeed, improved recognition of positive social cues after a one-week administration of the antidepressants citalopram or reboxetine predicts future treatment response (Shiroma et al., 2014; Tranter et al., 2009).

Mirtazapine is a commonly used serotonergic and noradrenergic antidepressant (Fawcett and Barkin, 1998) that decreases recognition of fearful faces (Arnone et al., 2009), without the initial increase typical of the selective serotonin reuptake inhibitor citalopram (Harmer and Cowen, 2013). A single dose of mirtazapine also enhances memory for positive emotional events (Arnone et al., 2009). At the neural level, mirtazapine decreases right amygdala-hippocampal and frontostriatal responses to fearful versus happy facial expressions, increases responses of the parietal cortex to a reward task, and attenuates responses of the cortical midline structures (CMS) during self-referential processing after a single dose (Komulainen et al., 2016; Rawlings et al., 2010; Vollm et al., 2006). Thus, mirtazapine may rapidly decrease processing of threatening stimuli and increase processing of positive or rewarding stimuli and influence self-related processing.

Here we used spoken emotional narratives to investigate antidepressant effects on brain responses in healthy volunteers. We assessed whether/how a single dose of mirtazapine influences neural responses to continuously recorded valence and arousal dimensions of spoken emotional narratives. Further, we assessed how mirtazapine influences dynamic functional connectivity associated with valence and arousal using seed-based phase synchronization (SBPS), a novel method, allowing us to assess dynamic functional connectivity between every voxel of the brain. We aimed to investigate the early effects of mirtazapine on instantaneously varying functional connectivity related to dynamic emotional stimuli, modeling complex and dynamic daily life emotional situations. We expected mirtazapine to decrease neural responses to negative events (negative valence) of the narratives in the emotional circuits of the brain.

\section{Methods}

\subsection{Subjects}

The participants were 30 healthy, native Finnish-speaking, righthanded volunteers aged 18-35 years. They were recruited via advertisement for university students and word of mouth. The participants were screened with the Structured Clinical Interview for DSM-IV Axis I Disorders (First et al., 2002) by an investigator (either psychiatrist or psychiatric resident) of the research group. Exclusion criteria included any current or life-time psychiatric disorder, current use of illicit drugs or excessive consumption of alcohol ( $>24 \mathrm{U} /$ week for men and $>16$ $\mathrm{U} /$ week for women), and use of antidepressants, antipsychotic agents, mood stabilizers, systemic corticosteroids, beta blockers, or benzodiazepines. The study was approved by the Ethics Committee of Helsinki and Uusimaa Hospital District and conducted in accordance with the Declaration of Helsinki. Written informed consent was obtained from each participant.

The participants were allocated into two groups to either receive a single dose of mirtazapine $15 \mathrm{mg}$ two hours prior to the fMRI or be scanned without mirtazapine as a control group in an open-label design. The study was originally designed as randomized, placebo-controlled and double-blind, but in contrast to earlier reports (Rawlings et al., 2010; Vollm et al., 2006), scanning of the first ten subjects revealed that plausible double-blinding was not possible due to sedative effects of mirtazapine. Consequently, the blinding was broken for both researcher and subject and the study was re-designed as an open-label protocol. During the open-label phase we excluded six participants from the mirtazapine group due to excessive sedation or sleeping during the fMRI (participant reported that she/he had fallen asleep during the task or was asleep after the task or response rate in the task was $<90 \%$ ). Data collection was continued until there were 15 subjects without excessive sedation in both groups ( 6 male, 24 female, mean age 24 years, SD 3.72). Thus, the final sample comprised 30 participants. There were no significant differences in age, comprehensive school grade point average $(p=0.926$ and $p=0.893$, respectively, in independent samples $t$-test) or gender ( $p=0.128$ in Fisher's exact test) between the excluded and included subjects.

As an estimate of the sedative effect of mirtazapine, a self-assessment of tiredness (on a likert scale from 1 to 5) was performed at baseline (assessment time 1, $2 \mathrm{~h}$ before the fMRI) and right before the fMRI (assessment time 2).

\subsection{Task and stimuli}

The task and stimuli are described in Fig. 1. During fMRI the participants listened to thirty 45-s spoken narratives describing unpleasant, neutral, and pleasant events, with ten stories for each category (Nummenmaa et al., 2014b). The recorded narratives were read by a neutral female voice that provided no prosodic cues for the affective significance of the story content. The time series of valence and arousal dimensions of the narratives were derived from a previous study (Nummenmaa et al., 2014b), where 18 healthy volunteers (12 females, age 19-30 years, mean age 24.4 years) listened to the narratives and rated their emotional feelings (Table 1 ). The ratings averaged across subjects formed the time-series that were used as predictors in the fMRI analyses. The narratives evoked strong emotional reactions and activated emotional circuits of the brain (Nummenmaa et al., 2014b).

Subjects were instructed to listen to the narratives as if they were listening to the radio or a podcast and to try to become immersed in the stories by imagining the described events vividly. Each narrative was preceded by a $5 \mathrm{~s}$ fixation cross and a $15 \mathrm{~s}$ text explaining the general 
A)

$5 s$

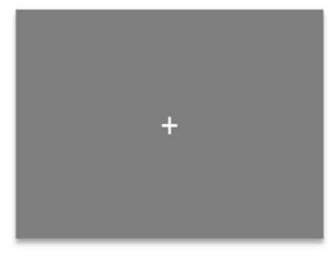

Fixation
$15 \mathrm{~s}$

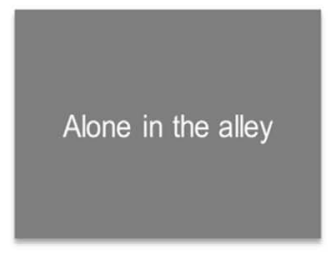

Text describing the general setting of the forthcoming story

B)

$45 \mathrm{~s}$

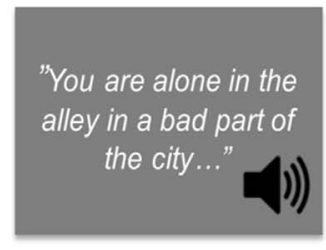

Story
$5 s$

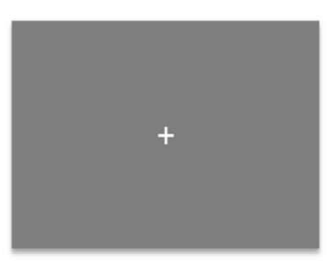

Fixation
Valence and arousal ratings

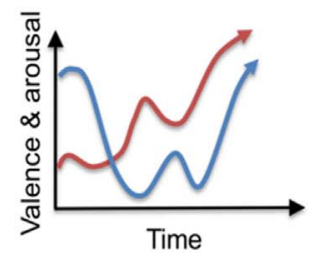

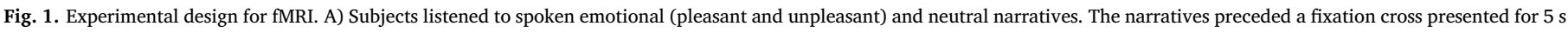

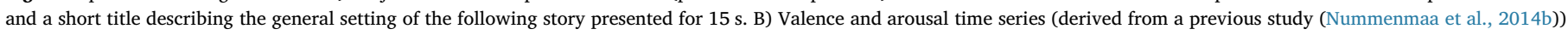
were used to assess neural responses to emotional content of the narratives.

Table 1

Mean valence and arousal ratings of the narratives (rescaled to range from 0 to 1 ). $\mathrm{UP}=$ unpleasant narratives, $\mathrm{PL}=$ pleasant narratives, $\mathrm{NE}=$ neutral narratives. A one-way analysis of variance (ANOVA) with Welch's F-test and Games-Howell's post hoc tests (due to inhomogeneity of variance) was used to compare the means.

\begin{tabular}{llllll}
\hline & $\begin{array}{l}\text { Mean UP } \\
(\mathrm{SD})\end{array}$ & $\begin{array}{l}\text { Mean PL } \\
(\mathrm{SD})\end{array}$ & $\begin{array}{l}\text { Mean NE } \\
(\mathrm{SD})\end{array}$ & $\mathrm{F}$ & $\mathrm{p}$ \\
\hline Valence & $\begin{array}{l}0.42 \\
\text { Arousal }\end{array}$ & $\begin{array}{l}0.78 \\
(0.11)^{\mathrm{a}, \mathrm{b}}\end{array}$ & $\begin{array}{l}0.66)^{\mathrm{a}, \mathrm{c}} \\
(0.05)^{\mathrm{b}, \mathrm{c}}\end{array}$ & $\begin{array}{l}\mathrm{F}(2,16.57) \\
=42.20\end{array}$ & 0.001 \\
& $0.62(0.10)^{\mathrm{b}}$ & $0.48(0.17)$ & $0.38(0.14)$ & $\begin{array}{l}\mathrm{F}(2,17.41) \\
=10.88\end{array}$ & 0.001 \\
\hline
\end{tabular}

\footnotetext{
${ }^{a}$ UP vs PL $\mathrm{p}<0.05$.

${ }^{\mathrm{b}} \mathrm{UP}$ vs NE $\mathrm{p}<0.05$

${ }^{c}$ PL vs NE $\mathrm{p}<0.05$.
}

setting of the forthcoming narrative without revealing its actual content. The latter epoch also served as a washout period for the emotion elicited by the previous narrative. During fMRI the stimuli were delivered using Presentation software (Neurobehavioral Systems Inc., Albany, CA, USA). The narratives were played to the subjects with an UNIDES ADU2a audio system (Unides Design, Helsinki, Finland) via plastic tubes through porous EAR-tip (Etymotic Research, ER3, IL, USA) earplugs. Sound was adjusted for each subject to be loud enough to be heard over the scanner noise.

\section{3. fMRI acquisition and statistical analysis}

Statistical analyses of baseline characteristics (age and comprehensive school grade point average) and the self-assessment of tiredness were performed with SPSS Statistics software, version 21 (IBM Corporation, Armonk, NY, USA). Independent samples $t$-test was used for baseline characteristics and repeated-measures analysis of variance (ANOVA) with group and assessment time as factors for the selfassessment of tiredness.

The MR imaging was performed on a 3T MAGNETOM Skyra wholebody scanner (Siemens Healthcare, Erlangen, Germany) at the Advanced Magnetic Imaging Center, Aalto NeuroImaging, Aalto University School of Science. Image acquisition has been described in detail previously (Nummenmaa et al., 2014b). Preprocessing and analysis were performed with SPM8 software (http://www.fil.ion.ucl. ac.uk/spm/). EPI images were realigned to the first scan by rigid-body transformation to correct head movements, co-registered and normalized to standard template (Montreal Neuroimaging Institute template) with linear and non-linear transformations and smoothed with Gaussian kernel of FWHM $8 \mathrm{~mm}$.

A random-effects model was implemented using a two-stage process. The first level (within-subject) general linear model (GLM) modeled the effect of valence and arousal parameters on BOLD response. The model included two explanatory variables, valence and arousal time series, and realignment parameters as effects of no interest to account for motion related variance. A high-pass filter of $128 \mathrm{~s}$ and AR(1) modeling of temporal autocorrelation were applied. Individual contrast images were generated for positive and negative effects of valence and arousal. In the second level models the resulting first-level contrast images were subjected to random effects analysis.

We first modeled the brain responses to the valence and arousal dimensions in the control group to track the neural correlates of valence and arousal without drug-effect by one-sample $t$-test. Next, the drugspecific effects were assessed by comparing the drug group and the control group by independent samples $t$-test. To quantify differences in sensory processing, the stories were modeled as boxcar functions and resulting contrast images were compared between the groups. The Statistical threshold was set at $p<0.05$, FDR corrected at cluster level.

We then assessed whether the sedative effect of mirtazapine confounds our results. We added the participants' self-assessment of tiredness (right before fMRI, i.e. assessment 2 ) as a covariate in the 2 nd level model (one-sample $t$-test) of the drug group separately.

\subsection{Static and dynamic functional connectivity}

Functional connectivity between different brain regions is usually measured time-independently, over the whole scanning session, assessing static synchrony between brain regions at rest or during specific tasks. In correlation-based connectivity methods it is possible to use a sliding time window to compute time-varying correlations, but higher temporal resolution comes at the expense of reliability of the results (Glerean et al., 2012; Sakoğlu et al., 2010). Recently it has been demonstrated that using phase synchronization as a measure of functional connectivity in fMRI data allows reliable computing of time-varying synchronization with a maximal temporal resolution (Glerean et al., 2012). Thus, seed-based phase synchronization (SBPS) enables assessing instantaneous changes in synchronization between brain regions evoked by complex, dynamic stimuli such as narratives or movies (Glerean et al., 2012; Nummenmaa et al., 2014b).

We estimated average functional connectivity using Pearson's correlation and instantaneous dynamic functional connectivity using seed-based phase synchronization (SBPS (Glerean et al., 2012), code available at: https://github.com/eglerean/funpsy). Because calculation of all possible voxel-wise connections $\left(\sim 3.5^{*} 10^{8}\right.$ connections) would be computationally prohibitive at native EPI data resolution, we spatially down-sampled the data to $6 \mathrm{~mm}$ isotropic voxels prior to estimating the time-variable functional connectivity. Voxels outside gray matter were masked out, resulting in $51836 \mathrm{~mm}$ isotropic voxels considered as functional nodes. This produced networks of $\sim 13$ million links. We first implemented further preprocessing steps following the recommendations of Power et al. (Power et al., 2014). Specifically, BOLD time series 
were band-pass filtered at $0.01-0.08 \mathrm{~Hz}$, head motion parameters were regressed (Friston expansion, 24 regressors), as were signals at white matter, ventricles, and cerebral spinal fluid. Global signal was not regressed. For each participant, a whole brain network was then computed as the pair-wise Pearson's correlation between all nodes time series. As a measure of group differences, for each link, we computed a two-sample $t$-test on the Fisher-Z-transformed correlation values. For the group statistic, mean frame-wise displacement was considered a regressor of no interest (Yan et al., 2013). Statistical significance and multiple comparison correction were assessed with permutations using a Network-Based Statistic (NBS) method (Zalesky et al., 2010). As there are positive and negative t-values, NBS returned a significance threshold for the positive and negative tail. The most conservative of the two thresholds was chosen (i.e. the maximum of the absolute value of the two thresholds).

For the dynamic functional connectivity, we computed time series of phase difference between pairs of voxels for each individual after band-pass filtering the BOLD signals at $0.04-0.07 \mathrm{~Hz}$ and Hilbert transform (schematic in Fig. S1, for details see Glerean et al. (2012)). To assess emotion-state specific effects of mirtazapine, we computed a two-sample $t$-test between the two groups for each link time series and each time point so that we obtained a group level dynamic network of tvalue link time series. Also in this case, to control for head motion confound, the instantaneous value of frame-wise displacement was used as a regressor of no interest. Finally, to separate the effects of valence and arousal, the data were divided into segments (high and low valence and high and low arousal). Valence and arousal were considered separately due to a possible correlation between the two dimensions. This produced four connectivity maps of link group differences significantly co-varying with valence and arousal. Control for multiple comparisons was done as in (Nummenmaa et al., 2014b) by using positive FDR (Storey and Tibshirani, 2003) with $\mathrm{q}<0.1$.

For visualization, all connectivity maps were summarized by grouping nodes into predefined anatomical regions (using AAL atlas). Only the $20 \%$ of hubs with highest degree centrality, and betweenness centrality at connection density of 10\% (Rubinov and Sporns, 2010), were included in the grouping. These maps thus show the connections that were most prominently modulated by mirtazapine. Finally, voxelwise average node degrees were stored into a node degree or 'hub' maps, where voxel intensities reflect how many connections from each voxel were statistically significantly modulated (either positively or negatively) by mirtazapine in the four different conditions. Since node degree maps are not statistical maps, the average 90th, 95th, and 99th percentile were considered to represent the degree of importance of each node in the four conditions (corresponding to mean node degree values of 174, 224 and 331).

\section{Results}

\subsection{Baseline characteristics}

No significant differences emerged between the groups in age, comprehensive school grade point average, or gender ( 3 males in both groups) (Table 2). In repeated measures ANOVA of the self-assessment

Table 2

Demographic characteristics and self-assessment of tiredness (Tiredness $1=$ self-assessment $2 \mathrm{~h}$ before fMRI, Tiredness 2 = self-assessment right before fMRI, likert scale $1-5$ ).

\begin{tabular}{|c|c|c|c|}
\hline & $\begin{array}{l}\text { Mean Mirtazapine } \\
\text { (SD) }\end{array}$ & $\begin{array}{l}\text { Mean Control } \\
\text { (SD) }\end{array}$ & $\mathrm{t}$ value (p) \\
\hline Age & $23.5(1.51)$ & $23.8(4.95)$ & $-0.22(0.825)$ \\
\hline $\begin{array}{l}\text { Grade point } \\
\text { average }\end{array}$ & $9.0(0.40)$ & $9.2(0.54)$ & $-1.52(0.140)$ \\
\hline Tiredness 1 & $1.87(0.74)$ & $2.07(0.88)$ & $-0.67(0.508)$ \\
\hline Tiredness 2 & $3.20(1.08)$ & $1.93(0.92)$ & $3.40(0.002)$ \\
\hline
\end{tabular}
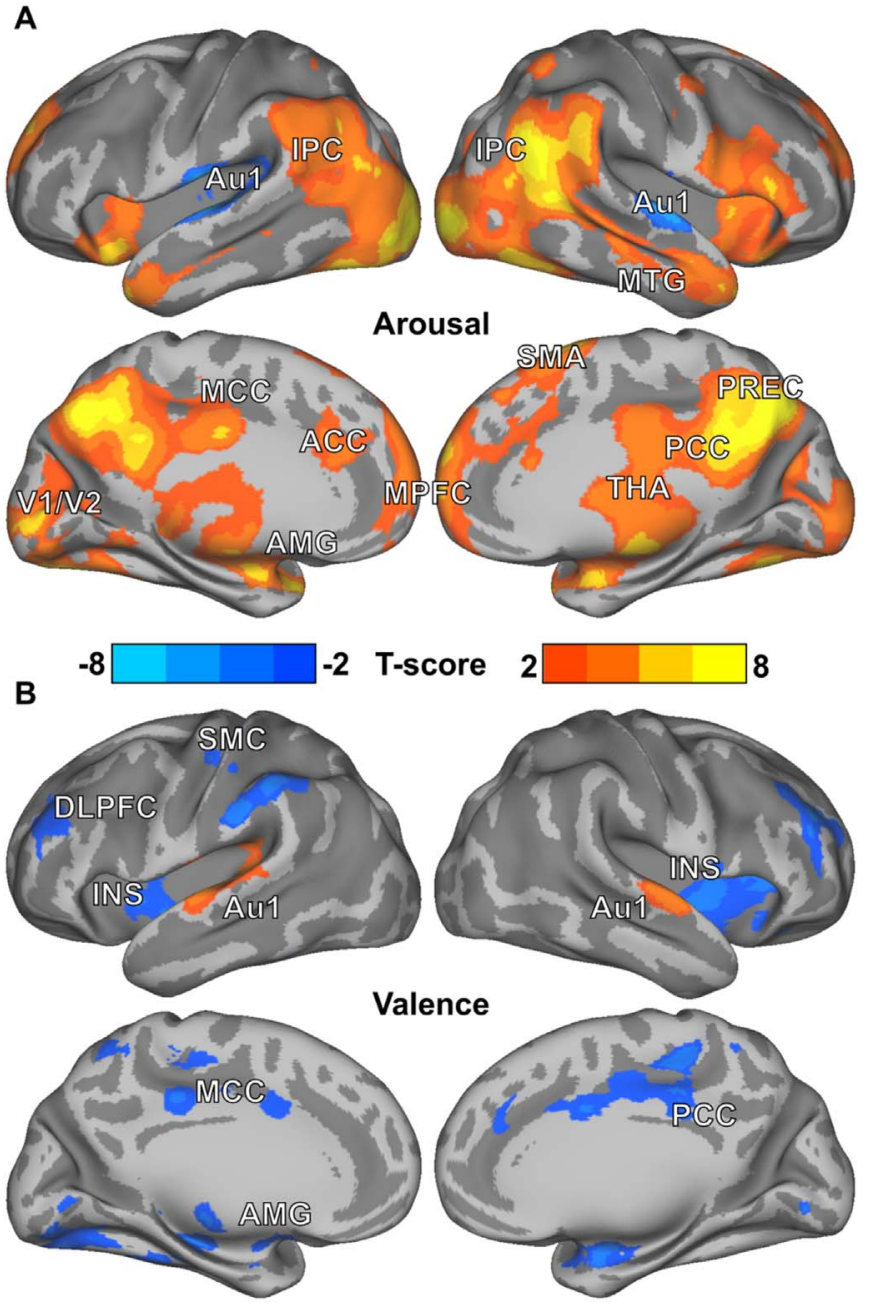

Fig. 2. Association of $\mathrm{A}$ ) arousal and $\mathrm{B}$ ) valence dimensions with BOLD signal in the unmedicated control group only $(\mathrm{p}<0.05$, FDR-corrected at cluster level). $\mathrm{AMG}=$ amygdala, $\mathrm{Au} 1=$ primary auditory cortex, $\mathrm{ACC}=$ anterior cingulate cortex, $\mathrm{DLPFC}=$ dorsolateral prefrontal cortex, $\mathrm{INS}=$ insula, $\mathrm{MCC}=$ middle cingulate cortex $\mathrm{MPFC}=$ medial prefrontal cortex, $\mathrm{MTG}=$ medial temporal gyrus, $\mathrm{PCC}=$ posterior cingulate cortex, $\mathrm{PREC}=$ precuneus, $\mathrm{SMA}=$ supplementary motor area, $\mathrm{SMC}=$ sensorimotor cortex, THA = thalamus, $\mathrm{V} 1=$ primary visual cortex, $\mathrm{V} 2=$ secondary visual cortex.

of tiredness there was a significant main effect of assessment time ( $F$ $(1,27)=9.54, p=0.005)$ and group*assessment time interaction $(F$ $(1,27)=14.67, p=0.001)$, but the main effect of group did not reach statistical significance $(F(1,27)=3.54, p=0.071)$. The mirtazapine group reported significantly increased tiredness at assessment 2 (Table 2).

\subsection{Regional effects}

There were no group differences in head motion (two-sample $t$-test on mean frame-wise displacement: $p=0.994$; two sample $t$-test on instantaneous frame-wise displacement not significant at $p<0.05$, FDR-corrected).

\subsubsection{Effect of valence and arousal in control group}

Results for the control group are summarized in Fig. 2 (for peak activations, see Table S1). Arousal was positively associated with increased activation in limbic areas (including amygdala, hippocampus and thalamus), striatum, primary and secondary motor areas, the cortical midline structures (CMS; MPFC extending to supplementary motor area (SMA), anterior cingulate (ACG), middle cingulate (DCG), posterior cingulate (PCG) and precuneus), occipital cortex (primary 
visual cortex and fusiform gyrus), temporoparietal regions (inferior parietal cortex (IPC), middle temporal gyrus and posterior superior temporal gyrus) and cerebellum. Valence was negatively associated with activation of the dorsolateral prefrontal cortex (DLPFC), limbic regions (including anterior insula, amygdala and hippocampus), DCG, PCG, somatomotor cortex (left primary (SI) and secondary (SII) somatosensory cortex and primary motor cortex), left IPC, occipital cortex (including lingual gyrus and right fusiform gyrus) and cerebellum. Activation in the auditory cortex (superior and transverse temporal gyri) was positively associated with valence and negatively associated with arousal.

\subsubsection{Effect of mirtazapine}

Whole-brain BOLD-GLM analysis revealed that mirtazapine reduced responses to arousing events of the stories in regions including bilateral amygdala-hippocampal complex, thalamus, CMS, visual, somatosensory (SI, SII), and motor cortices. Decreased responses to unpleasant events were observed in the right anterior insula and lateral and dorsolateral PFC. Overlapping effect of mirtazapine on the responses to high arousal and negative valence was seen in the ventromedial prefrontal cortex (VMPFC) and the ventral anterior cingulate cortex (vACC), visual, right somatosensory and motor cortices, temporoparietal cortex, and cerebellum (Fig. 3).

A
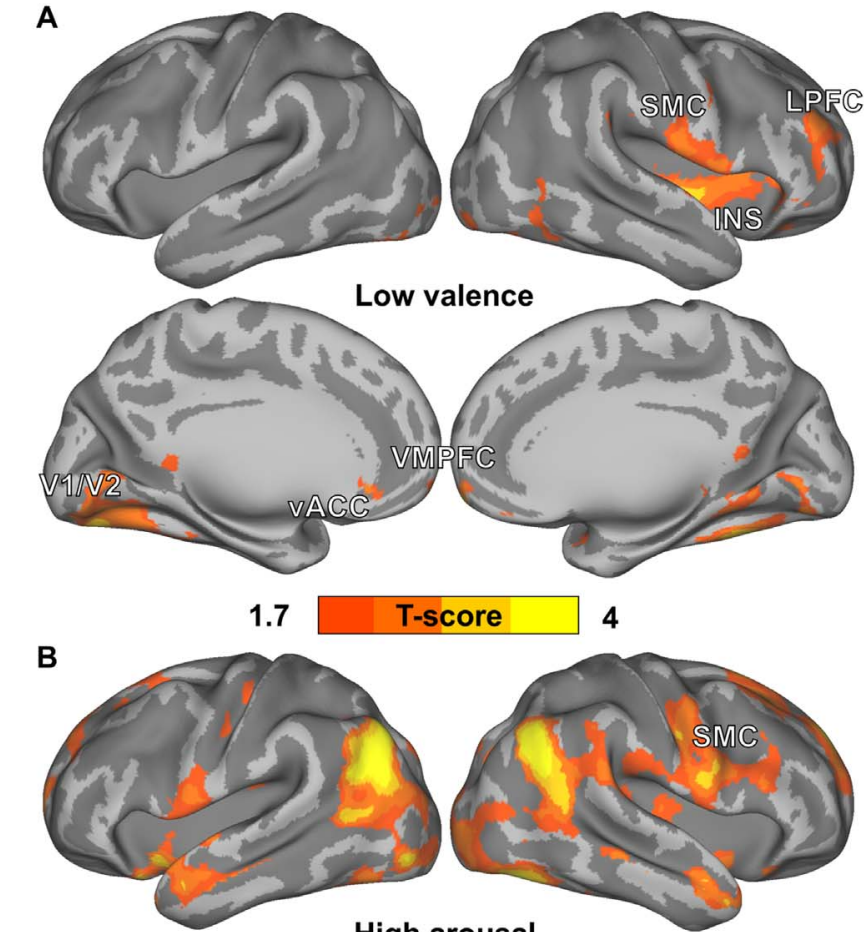

4

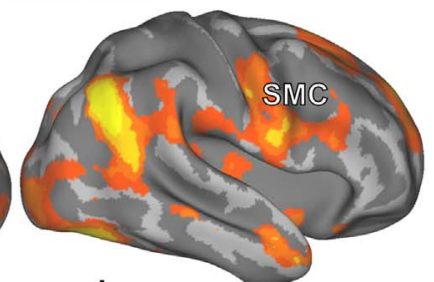

High arousal

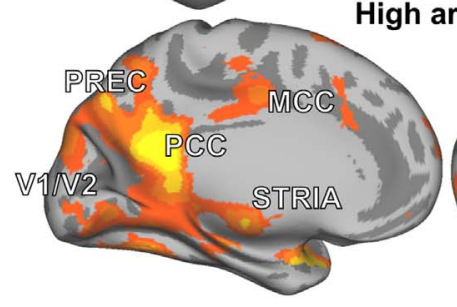

Fig. 3. Brain regions with significantly decreased activation in response to A) unpleasant events and B) arousing events of the narratives in the mirtazapine group relative to the control group. Statistical threshold at $\mathrm{p}<0.05$, FDR-corrected at cluster level. AMG $=$ amygdala, INS $=$ insula, $\mathrm{LPFC}=$ lateral prefrontal cortex, $\mathrm{MCC}=$ middle cingulate cortex, $\mathrm{MPFC}=$ medial prefrontal cortex, $\mathrm{PCC}=$ posterior cingulate cortex, $\mathrm{PREC}=$ precuneus, SMA = supplementary motor area, SMC $=$ sensorimotor cortex, STRIA = striatum, THA $=$ thalamus, vACC $=$ ventral anterior cingulate cortex, $\mathrm{VMPFC}=$ ventromedial prefrontal cortex, $\mathrm{V} 1=$ primary visual cortex, $\mathrm{V} 2=$ secondary visual cortex.
Adding subjective tiredness as a covariate in the medication group $t$ test of each contrast (valence and arousal) did not essentially change the results. There was no significant main effect of tiredness on responses of negative or arousing events of the stories in medication or control group. Critically, mirtazapine did not significantly decrease responses to the stories when they were modeled as boxcar functions without considering valence and arousal. In contrast, mirtazapine was associated with increased responses to the stories in the bilateral posterior hippocampus, posterior cingulate (PCG), and visual cortex.

\subsection{Effect on functional connectivity}

\subsubsection{Effect of mirtazapine on average functional connectivity}

Link-level connectivity differences across the whole experiment are summarized in Fig. S2 (see list of abbreviations in Table S2). Controls displayed on average higher connectivity values, with the most important differences involving functional connections in subcortical areas (thalamus, putamen, brainstem). The mirtazapine group showed increased connectivity between middle cingulate (DCG) and premotor areas (precentral gyrus, PreCG).

\subsubsection{Effect of mirtazapine on dynamic functional connectivity}

Of the networks of links significantly co-varying with the regressors, high-valence and low-arousal networks had the highest number of links for both control and mirtazapine groups (Table 3). Mirtazapine increased functional connectivity associated with high valence in the CMS (MPFC, ACG, DCG, PCG, and inferior parietal cortex (IPC)) and limbic regions (thalamus and hippocampus) - see Fig. 4 top right panel. This resulted primarily from increased connectivity between MPFC and DCG, MPFC and middle temporal gyrus, and IPC and DCG (see Fig. 5, bottom left panel). Mirtazapine decreased functional connectivity associated with high valence in somatosensory and motor cortices, DCG, and occipital regions (primary visual cortex, lingual gyrus, and fusiform gyrus) (Fig. 4 top right panel). This resulted from decreased connectivity between occipital and frontal areas, occipital and temporal areas, and occipital areas and DCG (Fig. 5, top left panel). Mirtazapine decreased functional connectivity associated with low valence mainly in the thalamus, striatum, fronto-insular cortex, and anterior CMS (MPFC, ACG, and DCG) and increased functional connectivity associated with low valence in the posterior CMS (PCG and precuneus).

Mirtazapine increased functional connectivity associated with low arousal in dorsofrontal areas (lateralized left), DMPFC, IPC, and occipital cortex, including lingual gyrus, fusiform gyrus, and parahippocampal gyrus (Fig. 4 bottom left panel, Fig. 5 bottom right panel). Mirtazapine decreased functional connectivity associated with low arousal in temporal areas, limbic regions (thalamus, hippocampus, and amygdala), VMPFC, and VACG, and occipital regions, including the primary visual cortex. Mirtazapine had virtually no effect on functional connectivity associated with high arousal.

\section{Table 3}

Summary properties of the networks composed of links co-varying with low/high valence/arousal for the two groups. Density is computed as significant number of links divided by total possible number of links in the network. Node degree is computed as number of significant links connected to a node.

\begin{tabular}{llllll}
\hline & \multicolumn{2}{l}{ Control group } & & \multicolumn{2}{l}{ Mirtazapine group } \\
\cline { 2 - 3 } \cline { 5 - 6 } \cline { 5 - 5 } & Density (\%) & $\begin{array}{l}\text { Node degree } \\
\text { (99th percentile) }\end{array}$ & & Density (\%) & $\begin{array}{l}\text { Node degree } \\
\text { (99th percentile) }\end{array}$ \\
\hline Low valence & 1.43 & 271 & 0.78 & 160 \\
High valence & 2.4 & 457 & 1.91 & 392 \\
Low arousal & 2.4 & 425 & 2.52 & 452 \\
High arousal & 0.79 & 182 & 0.84 & 184 \\
\hline
\end{tabular}



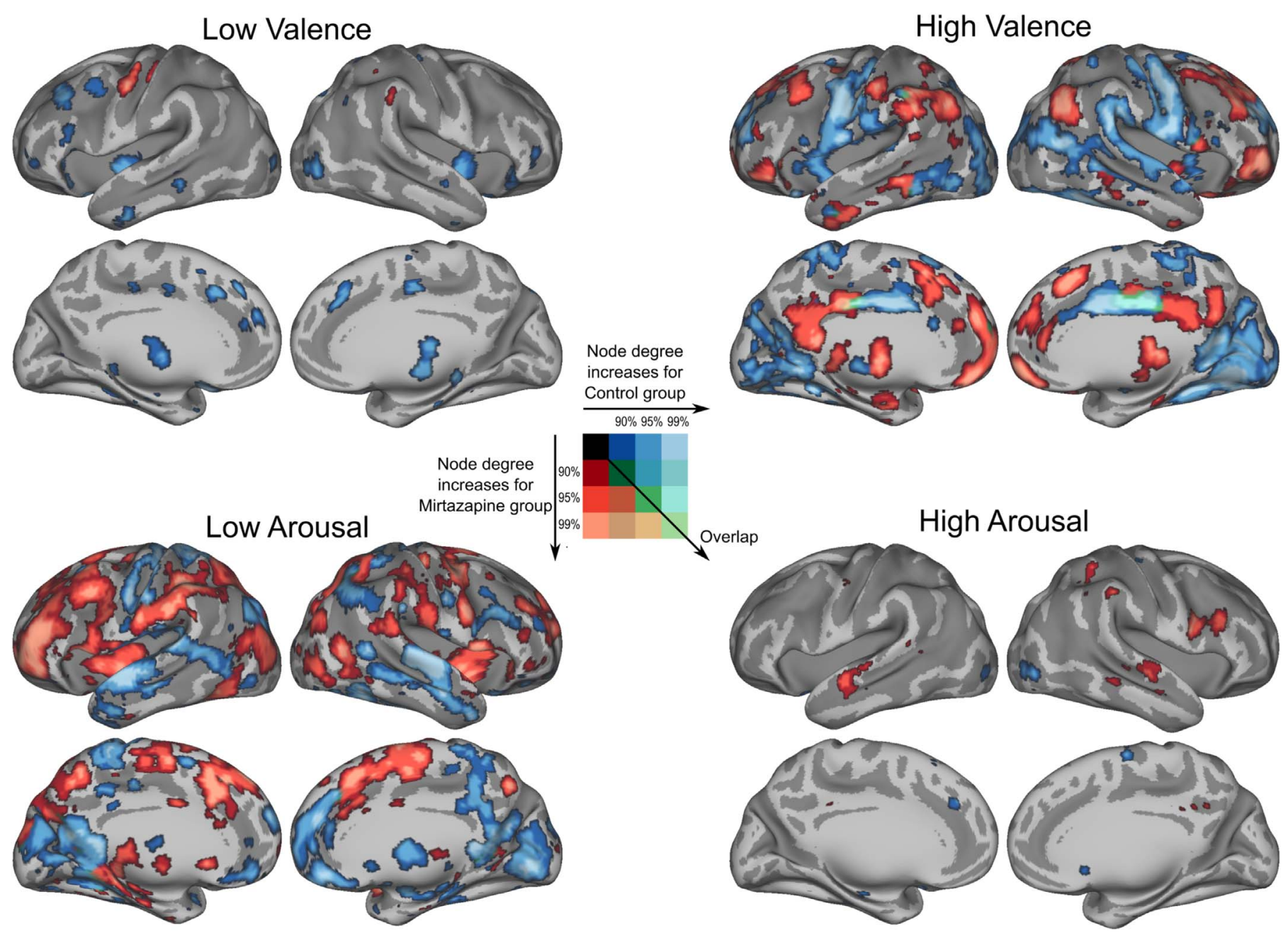

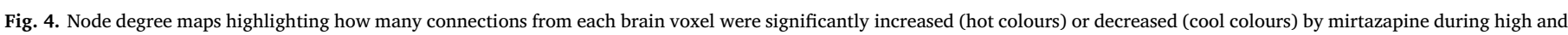
low valence and arousal episodes.

\section{Discussion}

A single dose of mirtazapine influenced neural responses and dynamic functional connectivity related to both valence and arousal dimensions of emotions while listening to spoken narratives lacking any prosodic cues to their emotional content. Previous antidepressant studies have used very simple emotional stimuli, most commonly briefly flashed emotional faces, and have mainly found locally altered activation, with attenuated limbic responses being the most consistent finding (Harmer and Cowen, 2013; Ma, 2015). Our results indicate that when using natural and complex emotional stimuli, and assessing not only regional responses but also dynamic functional connectivity, mirtazapine actually triggers large-scale changes spanning limbic, sensorimotor, and midline brain structures.

\subsection{Neural correlates of valence and arousal in the control group without medication}

In the control group, arousal was positively associated with activity of limbic and subcortical emotion circuits, cortical midline structures, temporo-parietal regions and visual and motor cortex. Valence was negatively associated with activation of limbic regions, DLPFC and sensorimotor cortex. This is in line with prior work showing that arousal and valence have distinct but partially overlapping neural basis, with arousal being associated with thalamus, insula, CMS and temporoparietal regions, and valence with core limbic regions including amygdala, insula and thalamus (Nummenmaa et al., 2014b). Also, previous studies using different sensory modalities as stimuli suggest that amygdala and thalamus may be important regions in tracking arousal dimension of emotions and frontal region in tracking valence dimension (Anderson et al., 2003; Colibazzi et al., 2010; Viinikainen et al., 2010).

\subsection{Effect of mirtazapine on arousal processing}

Mirtazapine decreased arousal-evoked responses of widespread neural circuits involved in arousal processing, including core emotional regions such as amygdala, thalamus, and striatum, the CMS, and sensorimotor cortices. The attenuating effect of mirtazapine on amygdala responses to threat as well as decreased recognition of subtle threatening stimuli have been observed in previous studies using simple stimuli and interpreted to reflect the anxiolytic effect of mirtazapine (Arnone et al., 2009; Rawlings et al., 2010). However, using natural and complex stimuli, closer to real-life emotional experiences, we observe a markedly larger-scale effect on arousal network.

It has been suggested that pathological anxiety may not be a disorder of fear only, but rather a disorder of hypervigilance (Davis and Whalen, 2001). Decrease in arousal-evoked responses of visual and motor cortices suggests that mirtazapine may immediately modulate vigilance, and consequently, actual sensory and motor responses to arousing events possibly via bottom-up connections from limbic regions to the neocortex. The CMS, such as DMPFC and DCG, have a role in motor preparation in response to emotional cues (Narayanan and Laubach, 2006; Oliveri et al., 2003; Vogt, 2005) and are implicated 


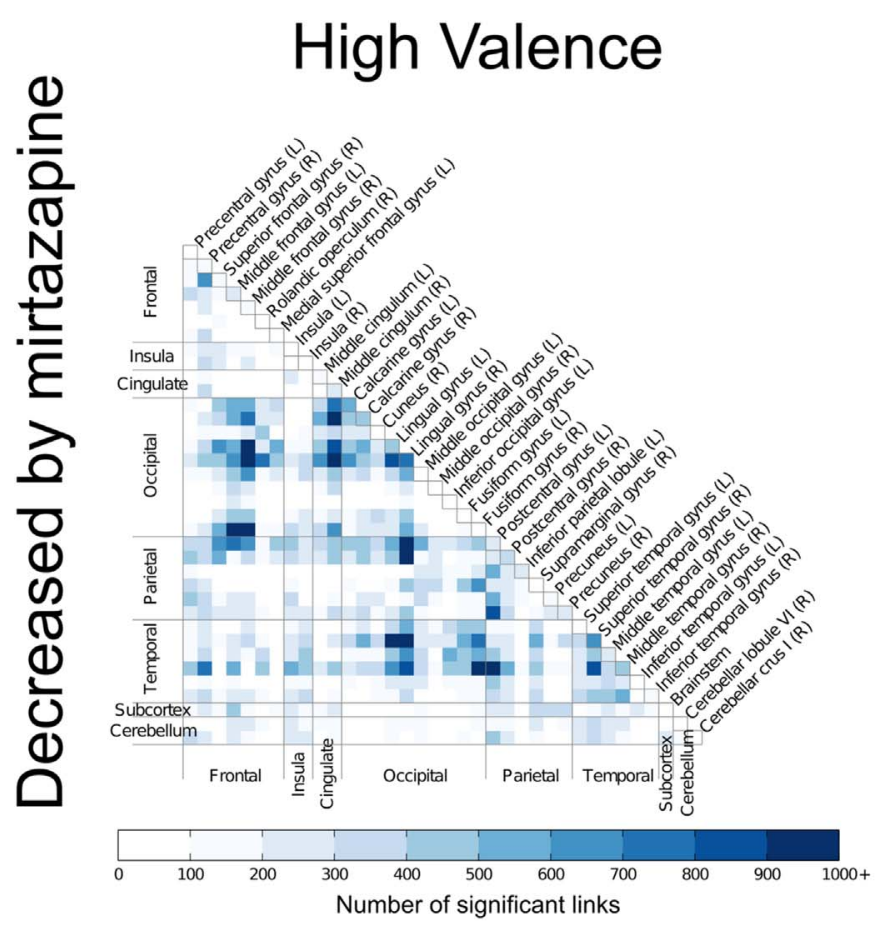

\section{Low Arousal}
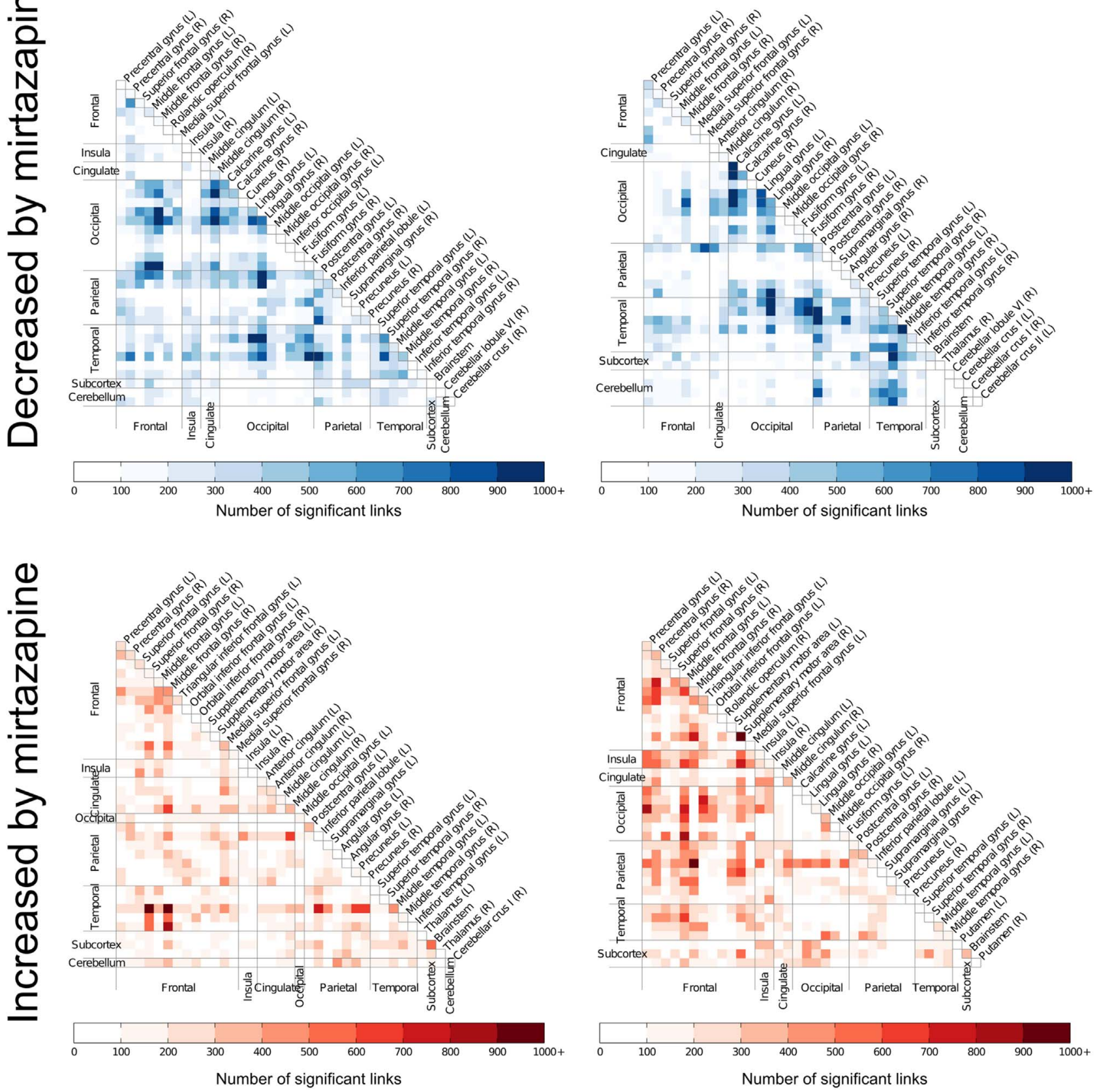

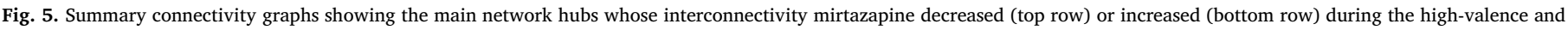
low-arousal narrative episodes. The reported value is the number of significant links between the two regions of interest (regions of interest based on the AAL atlas).

in avoidance behavior and fear processing (Oliveri et al., 2003; Vogt, 2005), receiving direct inputs from the amygdala (Vogt, 2005). Taken together, the attenuating effect of mirtazapine on the neural network tracking arousal dimension could possibly reflect the anxiolytic effect of mirtazapine, which in depressed patients may result in decreased sensory and behavioral responses to salient stimuli.

\subsection{Effect of mirtazapine on valence processing}

Mirtazapine decreased responses to unpleasant events of the stories in large-scale regions involved in valence processing, including right insula and DLPFC, sensorimotor cortices, and the anterior CMS
(VMPFC, vACG). Insula is implicated in affect perception and generation of affective state (Phillips et al., 2003), interoceptive awareness (Critchley et al., 2004), and subjective feelings (Phan et al., 2002). DLPFC has a role in higher-order, voluntary regulation of emotions (Phillips et al., 2008; Rive et al., 2013). Somatosensory cortex maps the bodily sensations associated with emotional stimuli (Nummenmaa et al., 2014a, 2008; Saarimäki et al., 2016), which constitute a core component of subjective emotional feelings (Nummenmaa et al., 2014a). The anterior CMS are also implicated in formation of subjective feelings (Saarimäki et al., 2016), as they are connected with the amygdala, primary sensory cortices, insula, midbrain, and brainstem, thus receiving exteroceptive and interoceptive information (Northoff 
and Sibille, 2014; Ongur and Price, 2000). These regions have a key role in self-referential processing, acting as a hub for tracking selfrelatedness of interoceptive and exteroceptive emotional signals (Lemogne et al., 2012). Our results suggest that mirtazapine may rapidly modulate neural circuits related not only to perception of unpleasant events, but also those responsible for generating subjective emotional feelings. Attenuation of the unpleasant subjective emotional feelings might significantly contribute to the therapeutic effect of mirtazapine. In line with this, MPFC and ACG are consistently implicated in the pathogenesis of depression (Drevets et al., 2008; Kaiser et al., 2015) and affected by antidepressant medication (Ma, 2015). Further, ACG appears to be the most promising region to predict treatment response of depressed patients across treatment modalities (Pizzagalli, 2011), suggesting this region as a key target for effective treatment interventions.

4.4. Effect of mirtazapine on large-scale functional connectivity associated with valence and arousal

Mirtazapine influenced large-scale dynamic functional connectivity related to valence and arousal dimensions of the stories. Mirtazapine specifically increased functional connectivity associated with positive valence and decreased functional connectivity associated with negative valence in the CMS and core emotional circuitries (including thalamus, hippocampus, insula, and striatum). Thus, mirtazapine was seen to potentiate processing of positive information in line with the previous findings of increased local responses to reward and simple positive cues after a single dose (Rawlings et al., 2010; Vollm et al., 2006). Our results suggest that mirtazapine may potentiate subjective experience and self-referential processing of positive information via increased cortical midline connectivity. However, mirtazapine decreased functional connectivity of visual and somatomotor cortices as well as the fusiform cortex associated with high valence. This may be related to the general negative effect of serotonergic antidepressants on affective experience sometimes reported (Price et al., 2009). Taken together, our results from the connectivity analyses support mirtazapine not only modulating local activity of single brain regions, but also modulating functions of large-scale brain networks implicated in the pathogenesis of depression (Drevets et al., 2008; Hamilton et al., 2012). These early changes may be important for the therapeutic effect of antidepressants. This is supported by a recent study that found changes in resting-state functional connectivity only $5 \mathrm{~h}$ after a single dose of escitalopram to predict later treatment response in depressed patients (Cheng et al., 2017).

\subsection{Limitations}

The sedative effect of mirtazapine can be argued to drive the observed changes in emotional responses. However, our control analyses do not support this; adding the subjective assessment of tiredness as a covariate in the medication group did not change the overall pattern of emotion-dependent brain responses. Moreover, brain responses to narratives per se (i.e. without modeling for affective features) did not differ between the groups in auditory cortices or cortical areas involved in semantic processing. This confirms that the sedative effect of mirtazapine did not interfere with or alter listening to and interpreting the narratives (Davis et al., 2007). The fact that mirtazapine did not significantly decrease neural responses to narratives per se also confirms that the group differences in responses to emotional content of the narratives was not merely due to some possible global effects of mirtazapine on BOLD signal, e.g. via vasodilatations. However, it must be kept in mind that the study is limited by its open-label design. Ideally, the effects of mirtazapine should be studied in a randomized, placebo-controlled, and double-blind design, yet the sedative effect of mirtazapine makes the blinding practically impossible (see Section 2). We investigated healthy volunteers, and thus, the generalizability of our findings to depressed patients requires further studies. We used a single dose of mirtazapine. The adaptive effects of continuous medications can lead to different results than those seen here.

\section{Conclusions}

Mirtazapine influences regional responses and dynamic functional connectivity of large-scale brain networks involved in encoding valence and arousal dimensions of emotions during natural conditions. We propose that these effects reflect the antidepressant and anxiolytic effects of mirtazapine, respectively. Mirtazapine reduced emotionevoked activity in the core affective (limbic) regions but also in sensory and motor cortices as well as the CMS. The CMS integrate sensory and emotional signals, and assess their self-relatedness, guiding appropriate motor, sensory, and autonomic responses (Duncan and Barrett, 2007; Murray et al., 2011; Northoff and Sibille, 2014). Changes in dynamic functional connectivity relative to the valence and arousal dimensions were observed between limbic, sensory, and cortical midline networks. These effects were observed when subjects listened to spoken narratives without prosodic emotional cues, meaning that higher-order cognitive and linguistic processing was required to extract the emotional content. Our results thus highlight that mirtazapine not only influences automatic, fight-or-flight responses to biologically salient sensory signals, but also modulates higher-level interaction between cognitive and affective processes during complex everyday events. The attenuation of responses to negative events, modulation of activity and connectivity in regions tracking self-relatedness of the events as well as increase of functional connectivity associated with positive events may all be relevant for the clinical effectiveness of this antidepressant.

\section{Contributors}

Authors EI, EK, JE, JL, KM, LN, PJ, TM and TTR designed the study. EK, KM and RH collected the data. EK, EG, JE and LN designed and performed the analyses. All authors contributed to and approved the final version of the manuscript.

\section{Conflicts of interest}

None.

\section{Acknowledgements}

We thank Marita Kattelus for her help with data acquisition. This work was supported by Academy of Finland (grant number 250935 to JE and MIND program grant number 265917 to LN) and ERC Starting Grant (number 313000 to $\mathrm{LN}$ ). The sponsors had no role in study design; collection, analysis and interpretation of the data; in the writing of the report; and in the decision to submit the article for publication.

\section{Appendix A. Supporting information}

Supplementary data associated with this article can be found in the online version at doi:10.1016/j.pscychresns.2017.03.009.

\section{References}

Anderson, A.K., Christoff, K., Stappen, I., Panitz, D., Ghahremani, D.G., Glover, G., Gabrieli, J.D., Sobel, N., 2003. Dissociated neural representations of intensity and valence in human olfaction. Nat. Neurosci. 6, 196-202.

Anderson, I.M., Del-Ben, C.M., McKie, S., Richardson, P., Williams, S.R., Elliott, R. Deakin, J.F., 2007. Citalopram modulation of neuronal responses to aversive face emotions: a functional MRI study. Neuroreport 18, 1351-1355.

Arnone, D., Horder, J., Cowen, P.J., Harmer, C.J., 2009. Early effects of mirtazapine on emotional processing. Psychopharmacology 203, 685-691.

Bigos, K.L., Pollock, B.G., Aizenstein, H.J., Fisher, P.M., Bies, R.R., Hariri, A.R., 2008. Acute 5-HT reuptake blockade potentiates human amygdala reactivity. 
Neuropsychopharmacology 33, 3221-3225.

Browning, M., Reid, C., Cowen, P.J., Goodwin, G.M., Harmer, C.J., 2007. A single dose of citalopram increases fear recognition in healthy subjects. J. Psychopharmacol. 21, 684-690.

Cheng, Y., Xu, J., Arnone, D., Nie, B., Yu, H., Jiang, H., Bai, Y., Luo, C., Campbell, R.A.A., Shan, B., Xu, L., Xu, X., 2017. Resting-state brain alteration after a single dose of SSRI administration predicts 8-week remission of patients with major depressive disorder. Psychol. Med. 47, 438-450.

Colibazzi, T., Posner, J., Wang, Z., Gorman, D., Gerber, A., Yu, S., Zhu, H., Kangarlu, A., Duan, Y., Russell, J.A., Peterson, B.S., 2010. Neural systems subserving valence and arousal during the experience of induced emotions. Emotion 10, 377-389.

Critchley, H.D., Wiens, S., Rotshtein, P., Ohman, A., Dolan, R.J., 2004. Neural systems supporting interoceptive awareness. Nat. Neurosci. 7, 189-195.

Davis, M., Whalen, P.J., 2001. The amygdala: vigilance and emotion. Mol. Psychiatry 6, 13-34.

Davis, M.H., Coleman, M.R., Absalom, A.R., Rodd, J.M., Johnsrude, I.S., Matta, B.F., Owen, A.M., Menon, D.K., 2007. Dissociating speech perception and comprehension at reduced levels of awareness. Proc. Natl. Acad. Sci. USA, 104, 16032-16037.

Del-Ben, C.M., Deakin, J.F., McKie, S., Delvai, N.A., Williams, S.R., Elliott, R., Dolan, M., Anderson, I.M., 2005. The effect of citalopram pretreatment on neuronal responses to neuropsychological tasks in normal volunteers: an FMRI study. Neuropsychopharmacology 30, 1724-1734.

Disner, S.G., Beevers, C.G., Haigh, E.A., Beck, A.T., 2011. Neural mechanisms of the cognitive model of depression. Nat. Rev. Neurosci. 12, 467-477.

Drevets, W.C., Price, J.L., Furey, M.L., 2008. Brain structural and functional abnormalities in mood disorders: implications for neurocircuitry models of depression. Brain Struct. Funct. 213, 93-118.

Duncan, S., Barrett, L.F., 2007. Affect is a form of cognition: a neurobiological analysis. Cogn. Emot. 21, 1184-1211.

Fawcett, J., Barkin, R.L., 1998. Review of the results from clinical studies on the efficacy, safety and tolerability of mirtazapine for the treatment of patients with major depression. J. Affect. Disord. 51, 267-285.

Ferstl, E.C., Rinck, M., von Cramon, D.Y., 2005. Emotional and temporal aspects of situation model processing during text comprehension: an event-related fMRI study. J. Cogn. Neurosci. 17, 724-739.

First, M.B., Spitzer, R.L., Gibbon, M., Williams, J.B.W., 2002. Structured Clinical Interview for DSM-IV-TR Axis I Disorders, Research Version, Patient Edition with Psychotic Screen. Biometrics Research. New York State Psychiatric Institute, New York.

Glerean, E., Salmi, J., Lahnakoski, J.M., Jaaskelainen, I.P., Sams, M., 2012. Functional magnetic resonance imaging phase synchronization as a measure of dynamic functional connectivity. Brain Connect. 2, 91-101.

Gotlib, I.H., Joormann, J., 2010. Cognition and depression: current status and future directions. Annu. Rev. Clin. Psychol. 6, 285-312.

Grady, C.L., Siebner, H.R., Hornboll, B., Macoveanu, J., Paulson, O.B., Knudsen, G.M., 2013. Acute pharmacologically induced shifts in serotonin availability abolish emotion-selective responses to negative face emotions in distinct brain networks. Eur. Neuropsychopharmacol. 23, 368-378.

Hamilton, J.P., Etkin, A., Furman, D.J., Lemus, M.G., Johnson, R.F., Gotlib, I.H., 2012. Functional neuroimaging of major depressive disorder: a meta-analysis and new integration of base line activation and neural response data. Am. J. Psychiatry 169, 693-703.

Harmer, C.J., Bhagwagar, Z., Perrett, D.I., Vollm, B.A., Cowen, P.J., Goodwin, G.M., 2003a. Acute SSRI administration affects the processing of social cues in healthy volunteers. Neuropsychopharmacology 28, 148-152.

Harmer, C.J., Cowen, P.J., 2013. 'It's the way that you look at it'-a cognitive neuropsychological account of SSRI action in depression. Philos. Trans. R. Soc. Lond. Ser. B Biol. Sci. 368, 20120407.

Harmer, C.J., Heinzen, J., O'Sullivan, U., Ayres, R.A., Cowen, P.J., 2008. Dissociable effects of acute antidepressant drug administration on subjective and emotional processing measures in healthy volunteers. Psychopharmacology 199, 495-502.

Harmer, C.J., Hill, S.A., Taylor, M.J., Cowen, P.J., Goodwin, G.M., 2003b. Toward a neuropsychological theory of antidepressant drug action: increase in positive emotional bias after potentiation of norepinephrine activity. Am. J. Psychiatry 160, 990-992.

Harmer, C.J., Mackay, C.E., Reid, C.B., Cowen, P.J., Goodwin, G.M., 2006. Antidepressant drug treatment modifies the neural processing of nonconscious threat cues. Biol. Psychiatry 59, 816-820.

Harmer, C.J., Shelley, N.C., Cowen, P.J., Goodwin, G.M., 2004. Increased positive versus negative affective perception and memory in healthy volunteers following selective serotonin and norepinephrine reuptake inhibition. Am. J. Psychiatry 161 1256-1263.

Kaiser, R.H., Andrews-Hanna, J.R., Wager, T.D., Pizzagalli, D.A., 2015. Large-scale network dysfunction in major depressive disorder: a meta-analysis of resting-state functional connectivity. JAMA Psychiatry 72, 603-611.

Komulainen, E., Heikkila, R., Meskanen, K., Raij, T.T., Nummenmaa, L., Lahti, J., Jylha, P., Melartin, T., Harmer, C.J., Isometsa, E., Ekelund, J., 2016. A single dose of mirtazapine attenuates neural responses to self-referential processing. J. Psychopharmacol. 30, 23-32.

Lemogne, C., Delaveau, P., Freton, M., Guionnet, S., Fossati, P., 2012. Medial prefrontal cortex and the self in major depression. J. Affect. Disord. 136, e1-e11.

Li, B., Liu, L., Friston, K.J., Shen, H., Wang, L., Zeng, L.L., Hu, D., 2013. A treatmentresistant default mode subnetwork in major depression. Biol. Psychiatry 74, 48-54.

Ma, Y., 2015. Neuropsychological mechanism underlying antidepressant effect: a systematic meta-analysis. Mol. Psychiatry 20, 311-319.

Murphy, S.E., Norbury, R., O'Sullivan, U., Cowen, P.J., Harmer, C.J., 2009. Effect of a single dose of citalopram on amygdala response to emotional faces. Br. J. Psychiatry.: J. Ment. Sci. 194, 535-540.

Murray, E.A., Wise, S.P., Drevets, W.C., 2011. Localization of dysfunction in major depressive disorder: prefrontal cortex and amygdala. Biol. Psychiatry 69, e43-54.

Narayanan, N.S., Laubach, M., 2006. Top-down control of motor cortices ensembles by dorsomedial prefrontal cortex. Neuron 52, 921-931.

Norbury, R., Mackay, C.E., Cowen, P.J., Goodwin, G.M., Harmer, C.J., 2007. Short-term antidepressant treatment and facial processing. Functional magnetic resonance imaging study. Br. J. Psychiatry.: J. Ment. Sci. 190, 531-532.

Norbury, R., Taylor, M.J., Selvaraj, S., Murphy, S.E., Harmer, C.J., Cowen, P.J., 2009. Short-term antidepressant treatment modulates amygdala response to happy faces. Psychopharmacology 206, 197-204.

Northoff, G., Sibille, E., 2014. Why are cortical GABA neurons relevant to internal focus in depression[quest] A cross-level model linking cellular, biochemical and neural network findings. Mol. Psychiatry 19, 966-977.

Nummenmaa, L., Glerean, E., Hari, R., Hietanen, J.K., 2014a. Bodily maps of emotions. Proc. Natl. Acad. Sci. USA, 111, 646-651.

Nummenmaa, L., Hirvonen, J., Parkkola, R., Hietanen, J.K., 2008. Is emotional contagion special? An fMRI study on neural systems for affective and cognitive empathy. NeuroImage 43, 571-580.

Nummenmaa, L., Saarimäki, H., Glerean, E., Gotsopoulos, A., Jääskeläinen, I.P., Hari, R., Sams, M., 2014b. Emotional speech synchronizes brains across listeners and engages large-scale dynamic brain networks. NeuroImage 102 (Part 2), 498-509.

Oliveri, M., Babiloni, C., Filippi, M.M., Caltagirone, C., Babiloni, F., Cicinelli, P., Traversa, R., Palmieri, M.G., Rossini, P.M., 2003. Influence of the supplementary motor area on primary motor cortex excitability during movements triggered by neutral or emotionally unpleasant visual cues. Exp. Brain Res. 149, 214-221.

Ongur, D., Price, J.L., 2000. The organization of networks within the orbital and medial prefrontal cortex of rats, monkeys and humans. Cereb. Cortex 1991, 206-219.

Phan, K.L., Wager, T., Taylor, S.F., Liberzon, I., 2002. Functional neuroanatomy of emotion: a meta-analysis of emotion activation studies in PET and fMRI. NeuroImage 16, 331-348.

Phillips, M.L., Drevets, W.C., Rauch, S.L., Lane, R., 2003. Neurobiology of emotion perception I: the neural basis of normal emotion perception. Biol. Psychiatry 54, 504-514.

Phillips, M.L., Ladouceur, C.D., Drevets, W.C., 2008. A neural model of voluntary and automatic emotion regulation: implications for understanding the pathophysiology and neurodevelopment of bipolar disorder. Mol. Psychiatry 13, 829-857.

Pizzagalli, D.A., 2011. Frontocingulate dysfunction in depression: toward biomarkers of treatment response. Neuropsychopharmacology 36, 183-206.

Power, J.D., Mitra, A., Laumann, T.O., Snyder, A.Z., Schlaggar, B.L., Petersen, S.E., 2014. Methods to detect, characterize, and remove motion artifact in resting state fMRI. NeuroImage 84, 320-341.

Price, J., Cole, V., Goodwin, G.M., 2009. Emotional side-effects of selective serotonin reuptake inhibitors: qualitative study. Br. J. Psychiatry.: J. Ment. Sci. 195, 211-217.

Rawlings, N.B., Norbury, R., Cowen, P.J., Harmer, C.J., 2010. A single dose of mirtazapine modulates neural responses to emotional faces in healthy people Psychopharmacology 212, 625-634.

Rive, M.M., van Rooijen, G., Veltman, D.J., Phillips, M.L., Schene, A.H., Ruhe, H.G., 2013. Neural correlates of dysfunctional emotion regulation in major depressive disorder. A systematic review of neuroimaging studies. Neurosci. Biobehav. Rev. 37, 2529-2553.

Rubinov, M., Sporns, O., 2010. Complex network measures of brain connectivity: uses and interpretations. NeuroImage 52, 1059-1069.

Saarimäki, H., Gotsopoulos, A., Jääskeläinen, I.P., Lampinen, J., Vuilleumier, P., Hari, R., Sams, M., Nummenmaa, L., 2016. Discrete neural signatures of basic emotions. Cereb. Cortex 26, 2563-2573.

Sakoğlu, Ü., Pearlson, G.D., Kiehl, K.A., Wang, Y.M., Michael, A.M., Calhoun, V.D., 2010. A method for evaluating dynamic functional network connectivity and taskmodulation: application to schizophrenia. Magn. Reson. Mater. Phys. Biol. Med. 23, $351-366$.

Shiroma, P.R., Thuras, P., Johns, B., Lim, K.O., 2014. Emotion recognition processing as early predictor of response to 8-week citalopram treatment in late-life depression. Int. J. Geriatr. Psychiatry 29, 1132-1139.

Storey, J.D., Tibshirani, R., 2003. Statistical significance for genomewide studies. Proc. Natl. Acad. Sci. USA, 100, 9440-9445.

Takahashi, H., Yahata, N., Koeda, M., Takano, A., Asai, K., Suhara, T., Okubo, Y., 2005 Effects of dopaminergic and serotonergic manipulation on emotional processing: a pharmacological fMRI study. NeuroImage 27, 991-1001.

Tranter, R., Bell, D., Gutting, P., Harmer, C., Healy, D., Anderson, I.M., 2009. The effect of serotonergic and noradrenergic antidepressants on face emotion processing in depressed patients. J. Affect. Disord. 118, 87-93.

Wallentin, M., Nielsen, A.H., Vuust, P., Dohn, A., Roepstorff, A., Lund, T.E., 2011 Amygdala and heart rate variability responses from listening to emotionally intense parts of a story. NeuroImage 58, 963-973.

Viinikainen, M., Jaaskelainen, I.P., Alexandrov, Y., Balk, M.H., Autti, T., Sams, M., 2010 Nonlinear relationship between emotional valence and brain activity: evidence of separate negative and positive valence dimensions. Hum. Brain Mapp. 31, 1030-1040.

Vogt, B.A., 2005. Pain and emotion interactions in subregions of the cingulate gyrus. Nature reviews. Neuroscience 6, 533-544.

Vollm, B., Richardson, P., McKie, S., Elliott, R., Deakin, J.F., Anderson, I.M., 2006. Serotonergic modulation of neuronal responses to behavioural inhibition and reinforcing stimuli: an fMRI study in healthy volunteers. Eur. J. Neurosci. 23 $552-560$.

Vrana, S.R., Lang, P.J., 1990. Fear imagery and the startle-probe reflex. J. Abnorm. Psychol. 99, 189-197.

Yan, C.-G., Cheung, B., Kelly, C., Colcombe, S., Craddock, R.C., Di Martino, A., Li, Q., Zuo, X.-N., Castellanos, F.X., Milham, M.P., 2013. A comprehensive assessment of regional variation in the impact of head micromovements on functional connectomics. NeuroImage 76, 183-201.

Zalesky, A., Fornito, A., Bullmore, E.T., 2010. Network-based statistic: identifying differences in brain networks. NeuroImage 53, 1197-1207. 